\title{
A highly flattened Secondary Star in the Young Eclipsing Binary BM Orionis*
}

\author{
DOUGLAS S. HALI (Nashville)
}

\begin{abstract}
A model is proposed for BM Ori to explain why DOREMUS saw only the early B star in the spectrum, whereas the light curve seemed to predict an equally bright cooler star which should have been seen alone at supposed totality. The cool star is assumed to be a disk-shaped object, similar in shape to that proposed by HUANG for $\varepsilon$ Aur, seen nearly edge-on. By means of various restraints, it is estimated that the disk covers about $55 \%$ of the B star at primary eclipse. This leads to the relative luminosities $\left(L_{h}=0.74,0.87\right.$ and 0.92 in $V, B$, and $U$ ), the color of the disk (the opposite side of the disk has the color of a $G$ or $F$ giant), the relative geometrical dimensions $\left(\mathrm{r}_{\mathrm{B}}=0.09, \mathrm{rD}_{\mathrm{D}}=0.25\right.$, and the height of the disk $\mathrm{h} \approx 0.1$ ), and the orbital inclination $\left(i \approx 90^{\circ}\right.$ ). The model with these parameters predicts a theoretical light curve which represents the observations as well as did the solution of HALI and GARRISON, which was based on spherical stars.

The mass-luminosity relation, in the form of IBEN's evolutionary tracks, is applied to the B 3 star and leads to the following absolute dimensions: $5 \mathfrak{N}_{\odot}$ for the $B 3$ star and $3 \mathfrak{N} \odot$ or $1 \mathfrak{2 N} \odot$ for the disk, depending on whether the mass function of STRUVE and TITUS or that of DOREMUS is used. The latter case corresponds nearly to the situation where the disk it at the Roche limit. The $B 3$ star has a radius of only $2.5 \mathrm{R}_{\odot}$; the disk is $15 \mathrm{R}_{\odot}$ in thickness.

A biconcave disk structure, such as calculated by BERLAGE for the primeval solar disk or by BODENHEIMER and OSTRIKER for pre-main-sequence stars undergoing rapid differential rotation, could satisfy the stringent requirement that the top and bottom of proposed disk appear exactly flat in projection.
\end{abstract}

\section{Introduction}

The eclipsing binary BM Orionis, the faintest of the four stars in the Trapezium, was the subject of a recent paper (HALL and GARRISON 1969). In that paper, referred to as Paper I, a photoelectric light curve was presented and solved and its implications discussed. It was argued that the primary eclipse, with its om7 depth und flat bottom, could not be annular and hence must be total. This led to the prediction that the cooler star, theretofore unseen in the spectrum (STRUVE and TITUS 1944), should be about as bright as the hot star and therefore should be visible in the spectrum outside eclipse as well as present along in the spectrum during the $8^{1 / 2}$ hours of supposed totality.

A serious paradox arose when DOREMUS (1970) obtained spectrograms which failed to reveal anything but the early B spectrum. One 18 minute exposure was taken at JD 2,440,524.026, two hours after the onset of totality, but only the B spectrum could be seen. In Paper I it was suggested that maybe the strong Balmer lines, not measured by STRUVE and TITUS because of the nebular emission, were produced in the photosphere of the cooler star, thought to be an A star. But DOREMUS measured them and found that their Doppler shifts also followed the radial velocity curve of the $B$ star.

Another difficulty which arose in Paper I was a very large apparent ultraviolet excess at the bottom of primary eclipse. If this eclipse was assumed total, one was forced to conclude that the cool star has an ultraviolet excess of $\delta_{\mathrm{U}}-\mathrm{V}=$ om7 !

In this paper a model is proposed which explains both of these problems and, if it is correct, shows that BM Ori is even more remarkable than might have been suspected.

In Paper I it was shown that, given the observed duration from first to fourth contact, about 16 hours, and the observed duration from second to third contact, about $8^{1 / 2}$ hours, the

\footnotetext{
* Reprints of the Arthur J. Dyer Observatory, Ser. XX, No. XX.
} 
ratio of the radii had to be 0.35 or less. Consequently, if primary eclipse were annular, its depth could be only about $12 \%$ of maximum light. Actually it was not pointed out in Paper 1 that limb darkening in the hot star could increase this depth somewhat, to $18 \%$ in the limit of total limb darkening; but since the depth of primary eclipse was observed to be mudh greater, $45 \%$ of maximum light, one would still reasonably conclude that the primary eclipse was total and, consequently, that the two components were of comparable luminosity.

To explain how the B star can be visible at the bottom of primary eclipse, the implicit assumption of spherical, or nearly spherical, stars is relaxed, and it is assumed that the cool component is a disk-shaped object seen nearly edge-on. This model is similar in shape to that proposed by HUANG (1965) to explain a similar paradox in $\varepsilon$ Aurigae.

For use in this paper the unrectified folded normal points in $V, B$ and $U$ are listed in Table I, since these were not published in Paper $\mathrm{I}$. Unit light corresponds to $\Delta \mathrm{V}=1 \mathrm{~m} 550$, $\Delta \mathrm{B}=\mathbf{1}^{\mathrm{m}} \mathbf{8 5 4}$, and $\Delta \mathrm{U}=2^{\mathrm{m}} \cdot \mathbf{2 4 2}$. Also given in Table II for convenience are the luminosities outside eclipse near the shoulders of primary minimum and near the shoulders of secondary minimum (the shoulders being different because of the reflection effect), at primary minimum, and at secondary minimum. Although given to three significant figures, the values could be uncertain by as much as 0.01 or 0.02 .

\section{The Model}

Whereas the B star is assumed essentially spherical, the cooler component is assumed to be a disk-shaped object, relatively faint and seen nearly edge-on. The geometry is illustrated in Figure 1; three cases are considered, corresponding to somewhat different orbital inclinations.

The proposed extreme flattening of the disk is not at all inconsistent with the very small $\mathrm{A}_{2}$ Fourier coefficient found in Paper $\mathrm{I}$. An $\mathrm{A}_{2}$ coefficient is produced by elongation in the direction of the line of centers but not at all by polar flattening. Furthermore, as will be shown later, the disk accounts for a rather small fraction of the total light.

From the observed phase angles of external and internal contact, $\Theta_{\mathrm{e}}=19^{\circ}$ and $\Theta_{\mathrm{i}}=10^{\circ}$, the dimensions in the orbital plane relative to the semi-major axis are seen to be approximately 0.25 and 0.08 . The 0.25 dimension is taken to be the relative radius of the disk, rD, while the 0.08 dimension is taken to be the relative radius of the B star, $\mathrm{rB}$. With this geometrical configuration the crucial aspects of the observed light curve can easily be explained. As the cooler disk passes in front of the B star it can produce the observed phase of constant brightness as a result of its flat top and bottom. In case I the top and bottom are seen in projection as straight lines; in cases II and III, as nearly straight lines. Also, it can produce the observed depth of primary eclipse yet allow the B star to be only partially eclipsed and hence dominant in the spectrum at mid-eclipse.

Although the calculations will not be presented here, it can be shown that the cool component cannot be a thin disk tilted. With the relative radius of only 0.25 , a thin disk could never eclipse more than about $15 \%$ of the area of the B star, where at least $45 \%$ is required. The additional area may not be supplied by a bulge resulting from a central star because such a bulge would spoil the flat edge needed to produce the observed flat bottom of primary eclipse.

From this point on it will be assumed that the model just proposed does in fact describe the binary BM Ori. In the next three sections various parameters of this assumed model will be evaluated in order to show that it can explain quantitatively the puzzling data at hand and is self-consistent in several respects.

\section{Luminosities and Colors}

Three simplifying assumptions will be made in the calculations which follow. First, zero limb darkening will be assumed for the B star so that there will be direct proportionality between area and luminosity. Second, although there is evidence of a reflection effect, it will not be handled in the usual way (RUSSELL and MERRILL 1952, § 44) because it is not certain if this would apply to such a strange object. For simplicity it will be assumed that each of the 

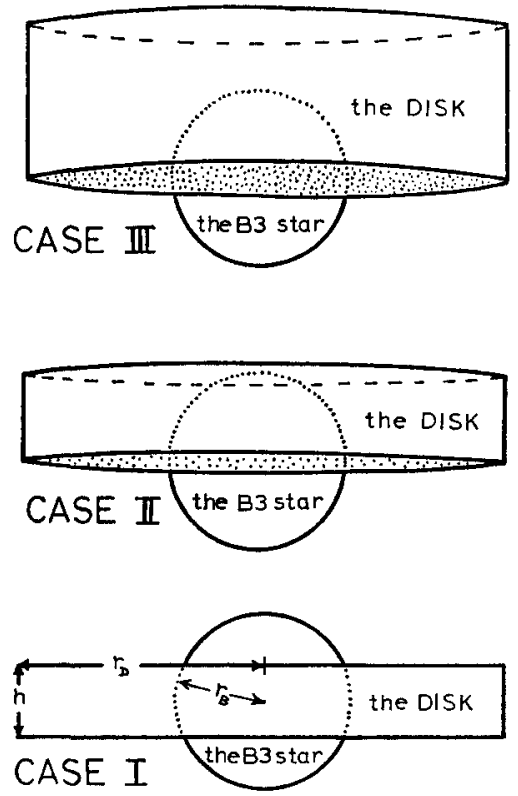

Fig. 1: The model proposed for the binary system BM Ori, as viewed from Earth at midprimary eclipse. The cool secondary component is a disk-shaped object. Primary eclipse appears to have a total phase because the top and bottom edges of the disk are seen in projection as straight lines - exactly straight in case $I$ and nearly straight in cases II and III. The bright B 3 star can be seen throughout primary eclipse because never more than about $50 \%$ is eclipsed.

two sides of the disk, the one facing the B star and the one opposite it, are uniformly bright although not equal in brightness to each other. Third, the very small ellipticity effect will be ignored.

The basic problem is to determine $\mathrm{f}_{\mathrm{B}}$, the fraction of the $\mathrm{B}$ star obscured at primary eclipse, and $f_{\mathrm{D}}$, the fraction of the disk obscured at secondary eclipse. It will be recalled that in the proposed model neither star is ever totally eclipsed by the other; therefore neither value will be unity. Furthermore the model is such that $\mathrm{f}_{B}$ and $\mathrm{f}_{\mathrm{D}}$ need to be equal, or even comparable.

First of all, however, it is possible to use the data in Table II to calculate the color of the light loss at primary eclipse (shoulder to minimum), which must logically be the color of the $B$ star. The resulting color indices, $\mathrm{B}-\mathrm{V}=+0 \mathrm{0m}, 5$ and $\mathrm{U}-\mathrm{B}=-\mathrm{om} 60$, are entirely independent of the value of $\mathrm{f}_{\mathrm{B}}$. As was shown in Paper $\mathrm{I}$, the colors are those of a B 3 star reddened by $\mathrm{E}(\mathrm{B}-\mathrm{V})=\mathrm{om} 3$. Although the color of the light loss at secondary eclipse should be the color of the facing side of the disk, the extreme shallowness of the eclipse, with its \pm 0.02 uncertainty in each color, leads to hopelessly uncertain color indices.

The procedure will be to assume a run of reasonable values of $\mathrm{fB}_{\mathrm{B}}$ and for each value to calculate the corresponding values of the relative luminosities of the components. Then three restraints will be imposed which will fix the value of $f_{B}$ quite precisely.

In all three colors the calculation of $\mathrm{L}_{B}, \mathrm{~L}_{D}{ }^{t}, \mathrm{~L}_{D}{ }^{\circ}$, and $\mathrm{F}_{\mathrm{D}}$ from each assumed value of $f_{B}$ was accomplished with the equations 
and

$$
\Delta \mathrm{I}_{0}^{\mathrm{prr}}=\mathrm{f}_{\mathrm{B}} \mathrm{L}_{\mathrm{B}}
$$

$$
\Delta \mathrm{l}_{\mathrm{o}}^{\mathrm{sec}}=\mathrm{fD}_{\mathrm{D}} \mathrm{L}_{\mathrm{D}}^{\mathrm{r}} \text {. }
$$

Here the symbol $L$ refers to relative luminosity, normalized to unity at maximum light, whidh is near the shoulder of secondary minimum where the B star and the facing side of the disk are completely in view. Near the shoulder of primary minimum, when the B star and the opposite side of the disk are completely in view, the light is somewhat less than maximum, as can be seen in Table II. The superscripts $f$ and $o$ refer to the facing and opposite sides of the disk. The quantities $\Delta l_{0}$ are the depths of each eclipse, in luminosity units, measured from the respective shoulders. The results are presented graphically in Figure 2. The Figure can be checked for consistency by noticing that for each color $L_{B}+L_{D}{ }^{t} \equiv 1$ for all assumed values of $f_{B}$.

The first restraint on $\mathrm{f}_{\mathrm{B}}$ is that the disk cannot be too bright. The disk, in the photographic or blue spectral region, must be faint enough that it can remain unseen in the spectrum. Specifically we can estimate that the opposite side of the disk must be at least one magnitude fainter than the fraction of the $B$ star which is uncovered at primary eclipse, $i$. e., $2.521 \mathrm{~L}_{\mathrm{D}}{ }^{0} \leq\left(1-\mathrm{f}_{\mathrm{B}}\right) \mathrm{L}_{\mathrm{B}}$. As can be seen in Figure 2 this corresponds to $\mathrm{f}_{\mathrm{B}}<0.57$. According to MURPHY (1971) this restraint should be relaxed somewhat because the rapid rotation expected for such a disk-shaped object would broaden its lines and render them difficult to detect in a spectrum.
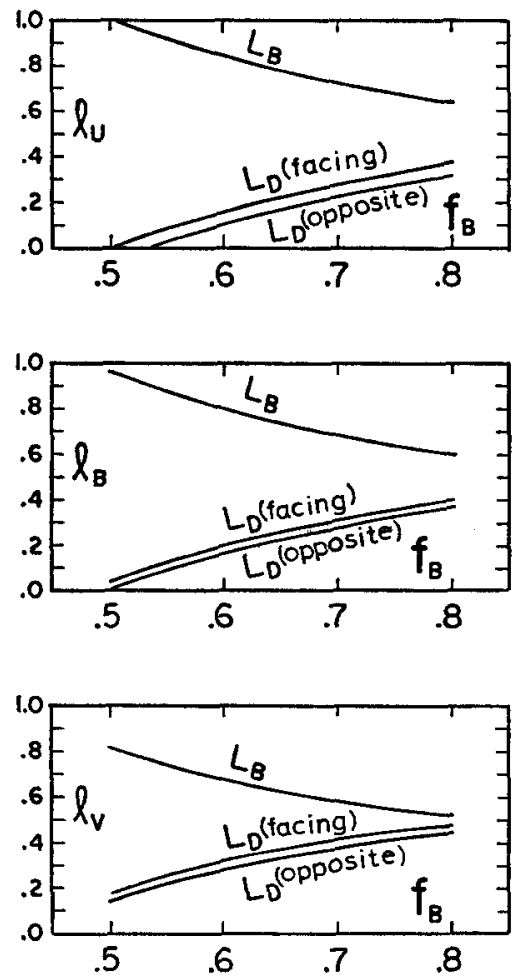

Fig. 2: The relative luminosities of the components of $\mathrm{BM}$ Ori in $\mathrm{V}, \mathrm{B}$, and $\mathrm{U}$ as a function of $\mathrm{f}_{\mathrm{B}}$, the fraction of the $B$ star obscured at primary eclipse. Notice that the sum $L_{B}+L_{D}$ (facing) is unity for all values of $f_{B}$. 


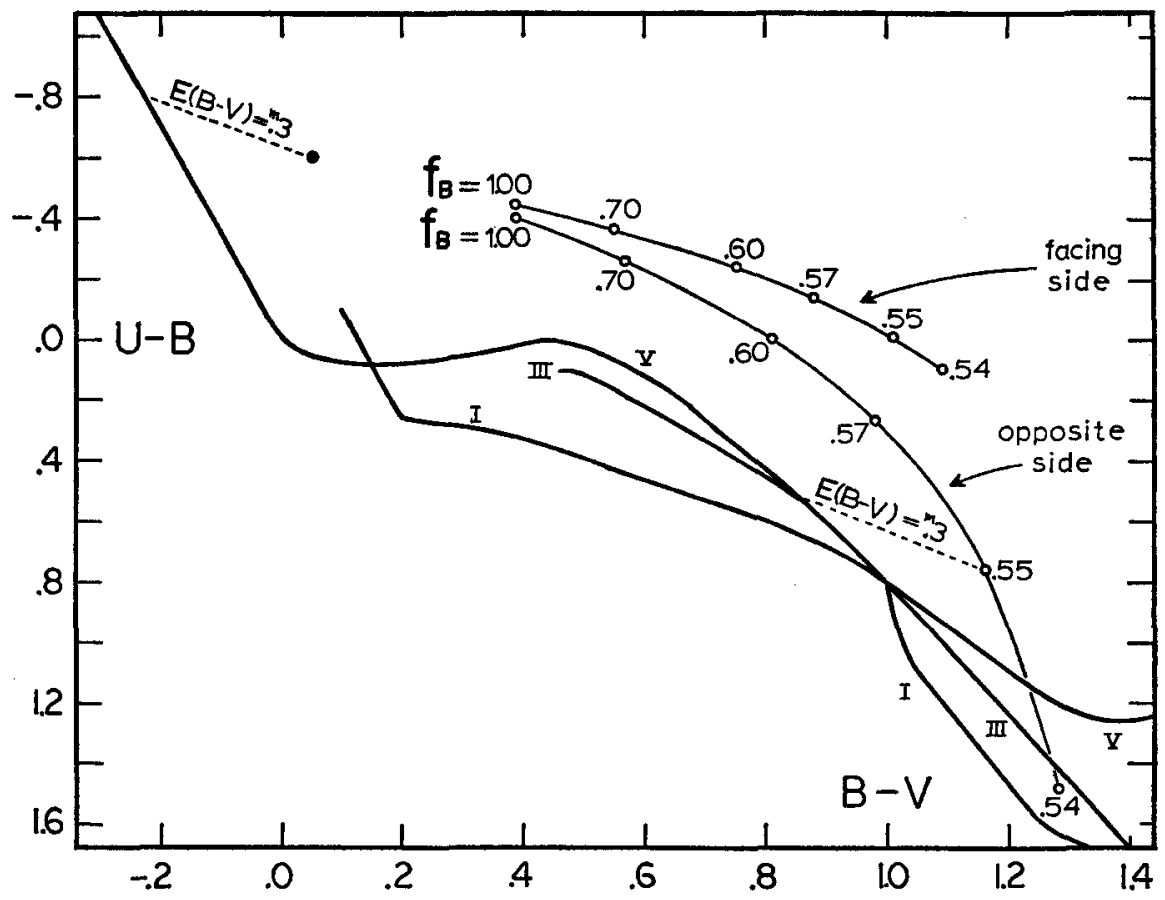

Fig. 3: The position of the individual components of BM Ori in the color-color diagram. The filled circle refers to the $B 3$ star, reddened by $E(B-V)=$ om3. The open circles refer to the two sides of the cool disk-shaped object, for various assumed values of $\mathrm{f}_{\mathrm{B}}$. Notice that if primary eclipse were total $\left(f_{B}=1\right)$ the cool star would appear to have a large ultraviolet excess. However if $\mathrm{fB}_{\mathrm{B}}=0.55$ the opposite side of the disk would have the color of a normal $G$ giant, reddened also by $E(B-V)=$ om. The colors of the facing side of the disk appear to be a mixture of red and blue light, presumably as a consequence of the reflection effect.

A second restraint on $\mathrm{f}_{\mathrm{B}}$ is that the luminosity of the disk not be negative. Examination of Figure 2 shows that, in order that $\mathrm{LD}_{D}{ }^{\circ}$ be positive in $\mathrm{U}, \mathrm{f}_{\mathrm{B}}>0.53$.

A third, very sensitive, restraint on $f_{B}$ is provided if we assume the $U-B$ and $B-V$ indices of the opposite side of the disk should be approximately those of a normal star with a reddening of $\mathrm{E}(\mathrm{B}-\mathrm{V})=$ om 3 . Figure 3 is a two-color diagram and shows the locus of colors implied for the disk when a run of values of $f_{B}$ is assumed. If $f_{B}=0.55$, then the opposite side of the disk is seen to have the colors of a G giant while the colors of the facing side of the disk appear to be a mixture of red and blue light, presumably as a consequence of the reflection effect.

It should be pointed out that there is a chance that the assumption of normal color indices for the opposite side of the disk may not be correct. Several cases are known in which the opposite side of the cool star in an eclipsing binary appears to have very large ultraviolet excess. Although the effect has yet to be explained satisfactorily, examples of it have been summarized recently by DEVINNEY, HALI, and WARD (1970, Table VIII).

On the basis of these three restraints, it seems reasonable to conclude that $f_{B}=0.55$ and to adopt this value in the subsequent discussion. The relative luminosities and $\mathrm{V}$ magnitudes resulting from this value of $\mathrm{f}_{\mathrm{B}}$ are presented in Table III. 
The spectral type of the disk in principle is determined by the last restraint, which is illustrated in Figure 3. However, since we are dealing with ratios of small numbers in evaluating color indices, realistic errors of a few percent in the light level at the bottom of primary eclipse would allow the implied spectral type of the disk to be as early as $\mathrm{F}$ and as late as $\mathrm{K}$. This would render the difference in visual magnitude between the disk and the B 3 star uncertain by about \pm om 3 , but would not appreciably affect the value $\mathrm{f}_{B}=0.55$.

A spectrogram taken recently by PARSONS (1971) in the infrared at $8500 \AA$ failed to reveal the secondary star. From this PARSONS has argued that the disk cannot be as late as $\mathrm{K}$ or $\mathrm{M}$ and therefore, in light of the limits provided by the UBV photometry, is probably of spectral type F or G. Since then MURPHY (1971) has obtained spectrograms, two in the same infrared regions and one in the blue. These also failed to reveal the secondary.

\section{Geometrical Dimensions}

In an attempt to determine the remaining geometrical elements, the total projected height of the disk $h$ and the orbital inclination $i$, three cases are considered, all of which succeed in producing the value $\mathrm{f}_{\mathrm{B}}=0.55$ which was required in the preceeding section. These three cases have already been illustrated approximately to scale in Figure 1, and their parameters are now listed in Table IV even though the detailed calculations are not presented here.

It can be seen from this table that the value of $\mathrm{f}_{\mathrm{D}}$ suffices to determine $\mathrm{h}$ and $\mathrm{i}$. The values of $L_{D}{ }^{r}$ given in Table III suggest that $f_{D} \approx 1 / 4$ because this value in equation 2 would result in depths of secondary minimum of $0.06,0.03$, and 0.02 in $V, B$, and $U$, which are consistent with the observed depths of $0.04,0.03$, and 0.04 , if one bears in mind that they could be uncertain by as much as 0.02 .

Thus it seems that BM Ori is described more nearly by case I or case II than by case III, that $\mathrm{h} \approx 0.1$, and that $\mathrm{i} \approx 90^{\circ}$. The model with these parameters is self-consistent in two respects, which can be pointed out at this time.

First, it can be seen in Figure 1 that this model will not allow more than about $1 / 3$ of the area of the disk to be covered by the $B 3$ star. The maximum value of $f_{D}$ occurs when $i=90^{\circ}$ and the disk is rather thin compared to the radius of the $B$ star, in which case

$$
\mathrm{f}_{\mathrm{D}} \approx \mathrm{rB}_{\mathrm{B}} / \mathrm{rD}=0.08 / 0.25=0.32 \text {. }
$$

Thus there is a logical restraint that $f_{D} \leq 0.32$. Because the deduced values of $L_{D}{ }^{p}$ in Table III and the observed depths of secondary minimum lead to a value of $\mathrm{fD}_{\mathrm{D}} \approx 1 / 4$, which satisfies this restraint, the model is indeed consistent.

Second, if case III had described the geometry, then the orbital inclination would have tilted the disk and caused its bottom edge to be seen in projection as a curved line. It can be shown that case IIl, which corresponds to $i=84^{\circ}$, would produce a curvature sufficient to make primary minimum about $6 \%$ deeper at mid-eclipse than at internal contact, $i$. e., $\Delta \mathrm{l}_{0}{ }^{\mathrm{pr} 1} / \Delta \mathrm{I}_{\mathrm{i}}^{\mathrm{pr} 1}=1.06$. It can be seen in Figure 4 (or more properly in Figure 3 of Paper $\mathrm{I}$ ) that the observations will not permit the depth to be more than a few percent deeper at mid eclipse, i. e., $\Delta_{0}{ }^{\mathrm{pri}} / \Delta_{i}{ }^{\mathrm{pr} 1} \lesssim 1.03$. This limit is nicely compatible with case I and case Il.

\section{Theoretical Light Curve}

It is possible to predict the shape the light curve of primary eclipse must have on the basis of this model and the parameters evaluated thus far. For the sake of definiteness we consider only case $\mathrm{I}$, and here again for simplicity we make the same three assumptions explained in the first paragraph of Section III. The shape was calculated, not with the familiar eclipsing binary tables of course, but by quite simple geometry, using equations similar to those HUANG (1965) used for the eclipse in $\varepsilon$ Aur. The resulting theoretical curve is presented in Figure 4 and there compared with the observed normal points which are taken from Table $I$.

For the ordinate in this figure it is convenient to use $n$, the fractional loss of light, taken to be zero at the shoulder of primary eclipse and normalized to unity at the bottom, because 


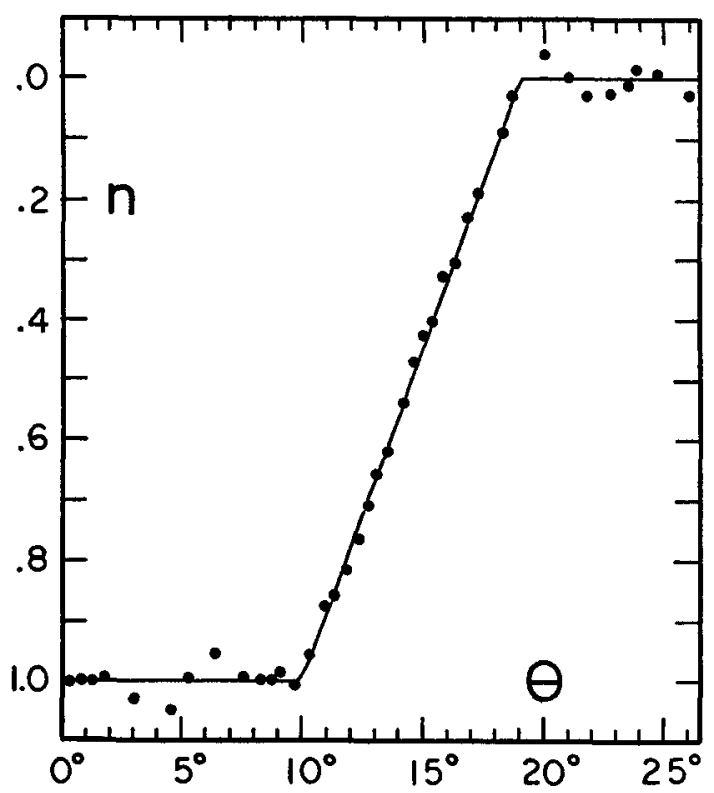

Fig. 4: The theoretical light curve predicted by case I of the proposed model with the values $\Theta_{\mathrm{i}}=9.8$ and $\Theta_{\mathrm{e}}=19.2$. This curve represents the observed normals, the filled circles, quite as well as did the solution in Paper 1 . Notice the very flat bottom, which seems to rule out cases II and III.

it is of interest to examine only the shape of the light curve. The best possible fit was achieved when the angles of internal and external contact were taken to be $\Theta_{i}=9 \%_{8}$ and $\Theta_{\mathrm{e}}=19{ }^{\circ}$. The resulting values of the relative radii were $\mathrm{rB}_{\mathrm{B}}=0.08$ and $\mathrm{rD}_{\mathrm{D}}=0.25$.

If a more realistic value such as $x=0.6$ had been assumed for the limb-darkening coefficient of the B star, the shape of the theoretical light curve would have been altered somewhat in the sense that a slightly smaller value of $\Theta_{\mathrm{i}}$ and larger value of $\Theta_{\mathrm{e}}$ would have been required to produce a fit of the same quality. A useful reference here is the bundle of light curves drawn by MERRILL (1953). The net effect would be to increase $\mathrm{IB}_{\mathrm{B}}$ and leave $\mathrm{rD}_{\mathrm{D}}$ unchanged. For this reason it was decided to use the value $\mathrm{rB}_{\mathrm{B}}=0.09$ in the calculation of absolute dimensions in the next section.

It is concluded that the present model predicts a theoretical light curve which satisfactorily represents the observed normal points. In fact if Figure 4 is compared with Figure 5 in Paper 1 , it can be seen that the disk model represents the observations quite as well as did the solution based on spherical stars, and perhaps a bit better.

\section{Absolute Dimensions}

The absolute dimensions of BM Ori were calculated in Paper I, but they were based

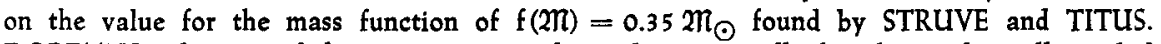
DOREMUS redetermined the spectroscopic orbit and unexpectedly found a much smaller radial velocity semi-amplitude, which resulted in a value of $f(\mathfrak{N})=0.02 \mathfrak{W} \odot$ ! 
One can only speculate now on the physical reason for the disagreement and guess which value of $f(\mathfrak{N})$ should be preferred. Therefore both values of $f(\mathfrak{N})$ will be considered here, as separate alternatives, in a redetermination of the absolute dimensions.

It is easy to estimate the absolute dimensions of BM Ori if we assume the B 3 star (which seems quite normal) is following an evolutionary track normal for its mass. It was argued in Paper I that one expects a binary as young as $10^{4}$ years still to be contracting towards the main sequence, but to avoid begging the question, both pre-main-sequence and post-mainsequence evolutionary tracks will be considered. The procedure, illustrated in Figure 5 , is to assume several values for the mass of the B 3 star, calculate its radius with Kepler's law and also with the evolutionary tracks, and see for which assumed mass the two different relations produce the same radius.

Kepler's law : for each assumed value of the mass of the $B 3$ star, $\mathfrak{N}_{B}$, the mass of the disk, $2 \mathfrak{N}_{D}$, can be calculated with either mass function, $f(\mathfrak{N})=0.02 \mathfrak{M}_{\odot}$ or $f(\mathfrak{N})=0.35 \mathfrak{M}_{\odot}$. Kepler's law then yields the semi-major axis of the orbit, $a$. The radius of the $B 3$ star then follows from the expression for the relative radius $\mathrm{r}_{\mathrm{B}}=\mathrm{R}_{\mathrm{B}} / \mathrm{a}=0.09$. The resulting relation between $\mathfrak{N}_{B}$ and $R_{B}$ is plotted in Figure 5 as a solid curve; actually there are two solid curves, one for each value of $f(\mathfrak{2})$ ).

Evolutionary tracks: It was shown earlier that the intrinsic color indices of the light loss at primary eclipse indicated a spectral type of B 3 for the hot star, in quite good agreement with the observed spectroscopic classifications. An effective temperature of $\mathrm{T}_{\text {eft }}=19,000^{\circ} \mathrm{K}$ will be adopted for this star (HARRIS 1963). An estimated uncertainty of about \pm 0 mo3 in the value of $(U-B)_{0}$ deduced for the $B 3$ star would render the value of $T_{\text {eff }}$ uncertain by about $1000^{\circ} \mathrm{K}$. In order to allow for additional uncertainty in the temperature scale itself, it is probably prudent to consider the value of $T_{\text {eff }}$ uncertain by about $2000^{\circ} \mathrm{K}$. Now for a given temperature and each assumed value of $2 \mathfrak{N}_{\mathrm{B}}$, the radius of the $\mathrm{B} 3$ star, $\mathrm{R}_{\mathrm{B}}$, can be read by interpolation in Figure 3 of IBEN (1965) or Figure 17 of IBEN (1967). The resulting relation between $\mathfrak{M}_{\mathrm{B}}$ and $\mathrm{R}_{\mathrm{B}}$ is plotted in Figure 5 for three values of $T_{\text {eff }}$, the dotted line referring to the pre-main-sequence case and the dashed line referring to the post-main-sequence case.

The following conclusions can be drawn from Figure 5. 1. The uncertainty in the value of $f(\mathfrak{N})$ has very little effect on the determination of the absolute dimensions derived for the B 3 star. 2. Likewise it makes very little difference whether pre- or post-main-sequence evolutionary tracks are used. 3 . The mass of the $B 3$ star is about $5 \mathfrak{2 N} \odot$ with an uncertainty of no more than $1 \mathfrak{N} \odot$. 4. the radius of the $B 3$ star is about $2.5 R_{\odot} \pm 0.3 R_{\odot}$ and is very close to the minimum radius predicted by IBEN's theoretical ZAMS (see PLAVEC 1968, eq. 1).

The absolute dimensions of the disk then follow from those of the B 3 star. The mass of the disk, however, is quite dependent on the mass function: $\mathfrak{M}_{\mathrm{D}}=3 \mathfrak{M} \odot$ if the value $f(\mathfrak{N})=0.35 \mathfrak{N}_{\odot}$ is used; and $\mathfrak{N}_{D}=1 \mathfrak{N}_{\odot}$ if the value $f(\mathfrak{N})=0.02 \mathfrak{N}_{\odot}$ is used. The relative radius $\mathrm{rD}_{\mathrm{D}}=0.25$ and the relative height $\mathrm{h} \approx 0.1$, lead to the absolute values of $7.5 \mathrm{R}_{\odot}$ and $3 \mathrm{R}_{\odot}$ respectively.

The absolute visual magnitude, $M_{\nabla}$, of the $B$ star was calculated from its radius of 2.5 $\mathrm{R}_{\odot} \pm 0.3 \mathrm{R} \odot$, its effective temperature of $\mathrm{T}_{\text {eff }}=19,000^{\circ} \mathrm{K} \pm 2000^{\circ} \mathrm{K}$, and the bolometric correction corresponding to this temperature (HARRIS 1963). The result is $M_{V}=-0 m 7 \pm 0 m 3$, where the uncertainty is caused primarily by the uncertainty in the radius, surprisingly little by the uncertainty in the effective temperature. This is because $T_{\text {eff }}$ and B.C. must be taken from HARRIS as a pair, enter into the above equation with opposite sign, and consequently have little effect on the visual magnitude. The $M_{v}$ of the disk can be calculated simply from the $M_{\mathrm{v}}$ of the Bstar and the difference in apparent visual magnitude, taken from Table III.

All of these absolute dimensions are summarized in Table V.

\section{Discussion}

The very small radius of the $B 3$ star, only $2.5 R_{\odot}$, may be the key in explaining why, according to MORGAN (JOHNSON 1965), the early stars in the innermost regions of the Trapezium cluster seem fainter than those in the region further out. Spectroscopically they 


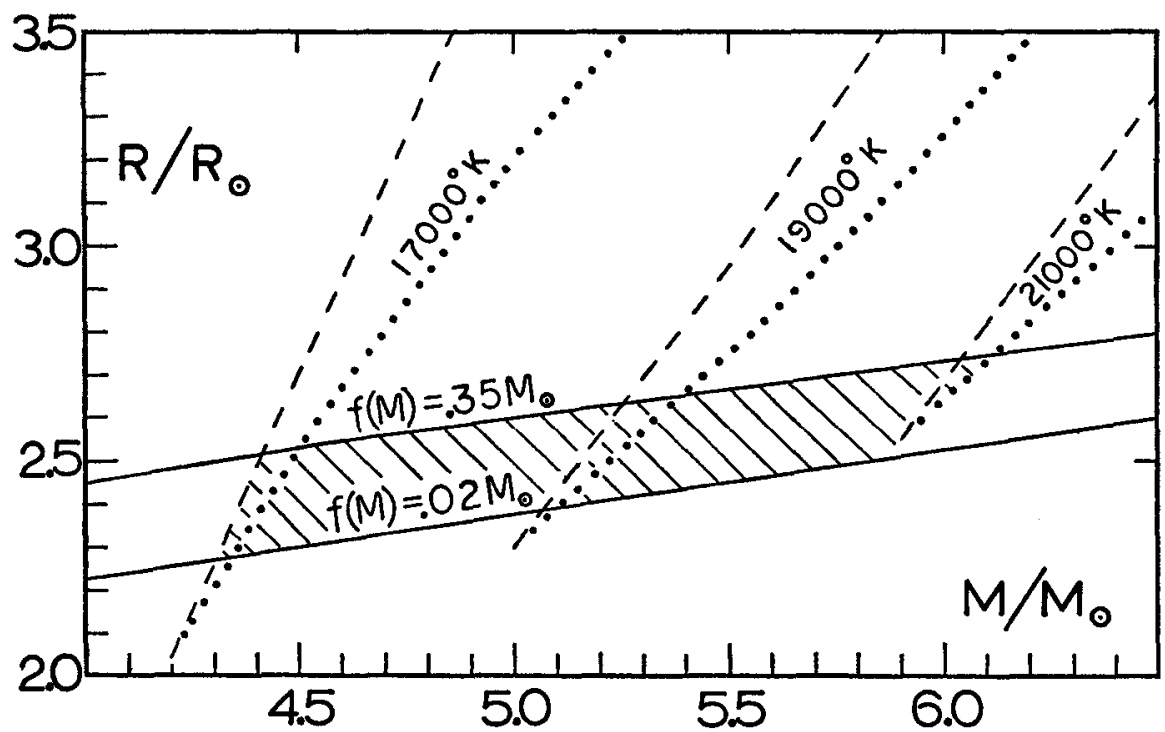

Fig. 5: The absolute dimensions of the B 3 star should lie within the shaded portion. The nearly horizontal solid lines result from Kepler's law and two possible values of $f(2)$ ). The diagonal lines result from the mass-luminosity relation of IBEN (pre-main-sequence dotted, post-main-sequence dashed) for three assumed values of $T_{\text {eff }}$ for the $B 3$ star, and all end at the minimum radius which defines the theoretical ZAMS.

seem fainter (broad $\mathrm{H}$ lines) and photometrically they are fainter ( $9 / 4$ of a magnitude on the average). Stars towards the center are probably younger and therefore more likely to have radii very near the minimum radius, which only extremely young stars have.

The disk-shaped object probably satisfies the rather stringent requirement of semi-stability described by HUANG (1963). Thus it may be that the thickness, or some other physical property, of the disk fluctuates somewhat on a time scale of a year or so. This could explain a curious effect which was pointed out in Paper I. The photometry of WALKER (1969) resulted in appreciably different magnitudes and colors outside eclipse and at the bottom of primary eclipse, but in such a manner that the color of the light loss at primary eclipse, which must logically be the color of the B star, was very closely that found by HALL and GARRISON. The implication is that the photometry in both years was quite accurate but that the dimensions and/or luminosity of the disk were somewhat different in 1958 than they were around 1968.

It happens, perhaps coincidentally, that the mass function of DOREMUS corresponds to a limit below which the disk would overflow its Roche lobe. The smaller value of $1 \mathfrak{N} \odot$ for the mass of the disk leads to a mass ratio of $q=0.2$ which, according to PLAVEC and KRATOCHVIL (1964), produces a radius of $b=0.24$ for the Roche lobe; the corresponding dimension of the disk, its radius in the orbital plane as seen at primary eclipse, is $r_{d}=0.25$. Until we can decide which mass function is correct, we cannot decide whether or not the disk fills its Roche lobe. However there is no evidence at present to suggest mass transfer, which usually occurs in semi-detached Algol-like binary systems (BATTEN 1970, PLAVEC 1970). Neither STRUVE and TITUS nor DOREMUS noticed any emission lines which could be attributed to circumstellar material around the B 3 primary. And the orbital period seems to have remained remarkably constant since the variability of BM Ori was discovered in 1917. 
This can be seen in Table VI where all observed times of primary minimum are listed along with the $\mathrm{O}-\mathrm{C}$ residuals computed with the new ephemeris given in Paper I

$$
\mathrm{JD} \text { (hel.) }=2,440,265.343+6.470525 \cdot \mathrm{E} \text {. }
$$

The first six times have been taken from PARENAGO (1947). PARENAGO also computed the time of conjunction from the radial velocity curve of STRUVE and TITUS and used it as a time of primary minimum; but because the spectroscopic orbit implies a large orbital eccentricity $\left(e=0.14, \omega=194^{\circ}\right)$ which is not consistent with the undisplaced secondary minimum found in Paper $l$, this computed time of conjunction has been considered suspect and is not listed in Table VI. The rising branch of primary eclipse observed by WALKER (1969) was compared with the complete light curve in Paper I to yield a time of minimum. It can be seen in Table VI that there is no indication of any systematic trend in the $\mathrm{O}-\mathrm{C}$ residuals, and that the only rather large residual is that of HAAS, which was considered by PARENAGO as uncertain.

It is informative to use the absolute dimensions in Table $V$ to estimate the age of the B 3 star in BM Ori with the pre-main-sequence evolutionary tradks of IBEN (1965). On the other hand it seems unwise to try to estimate the evolutionary age of the disk since it is uncertain how the physical nature of such an object should be imagined. The B 3 star, having nearly reached the minimum radius on the theoretical ZAMS, has an evolutionary age of about $5 \times 10^{5}$ years, considerably older than the age of $10^{4}$ years supposed for the Trapezium. This somewhat surprising result was pointed out in Paper I. Three possible explanations should be explored. 1. One possible explanation, offered in Paper $\mathrm{I}$, is based on the contention that evolutionary ages of binaries probably should not correspond to those calculated for single stars. 2. Since the $10^{4}$ year age of the Trapezium discussed by SHARPLESS (1966) is essentialiy an expansion age, it might be that the expansion began some time after the stellar formation began. 3. The evolutionary times of IBEN might have to be revised. Such a revision for massive stars has already been suggested by LARSON (1969) and is in the right direction, but it is not large enough to explain the discrepancy between $5 \times 10^{5}$ years and $10^{4}$ years.

It may be possible to find examples in theory of the severe flattening required by the model proposed in this paper. One point should be made very clear: the observed light curve requires that the top and bottom should appear almost exactly flat when seen in projection; it is not enough that the object be lenticular in shape because then its top and bottom would appear quite curved, even if seen exactly edge-on. Although the internal structure of the disk is unknown, let us consider two extremes. At one extreme there could be a central star, possessing most of the mass, imbedded in an optically thick disk, reminiscent of the primeval solar disk. According to BERLAGE (1968) the primeval solar disk was not lenticular in shape, but had a torus-like structure. This can be seen in his Fig. 20. At the other extreme the disk could be a relatively homogeneous object which will not dissipate but rather will slowly go to forming a spherical star as it redistributes or sheds angular momentum. Models of pre-main-sequence stars undergoing rapid differential rotation were calculated recently by BODENHEIMER and OSTRIKER (1970). As can be seen in their Figure 5, their configurations also develop a toruslike shape. Actually neither the primeval solar disk of BERLAGE nor the configuration of BODENHEIMER and OSTRIKER is a torus (there is no hole in the doughnut). They are better described as biconcave disks (shaped like red blood cells). Such an object is illustrated schematically in Figure 6, where the following conclusion can be drawn: when the disk is seen edge-on, the concavity guarantees that the top and bottom will appear exactly flat in projection.

An important question which might be answered now is why the secondary star is severely flattened but the primary star is not. Recently, MARKS and CLEMENT (1971) have examined the time scale with which differentially rotating stars adjust to radiative and viscous stresses as well as viscous diffusion. They find that for $B$ stars equilibrium is reached at the surface in a mere 1000 years, whereas for a star of $2.5 \mathfrak{M}_{\odot}$ the time scale is an order of magnitude longer. In BM Ori, thought to be $10^{4}$ years old, it looks as if the more massive B star has already reached equilibrium and taken on a normal shape, while the less massive secondary 


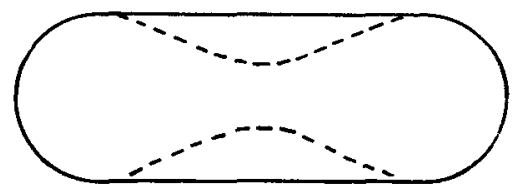

Fig. 6: A biconcave disk, such as calculated by BODENHEIMER and OSTRIKER (1970), seen edge-on. The dashed line is the cross-section. The concavity guarantees that the top and bottom will appear exactly flat in projection.

is still in the process of reaching equilibrium and is seen today with quite an unusual shape. Furthermore the time scales of MARKS and CLEMENT explain why one should not expect to see such highly flattened objects among any but the very youngest stars.

BM Ori, although an eclipsing binary, has its place in a colloquium on intrinsic variables because, as already mentioned, there is suspicion that the disk-shaped objects is unstable and varies in shape and/or luminosity. But of more importance, BM Ori forces us to admit that recently formed binaries need not arrive on the main sequence with both components completely normal. Therefore we should anticipate possible complications in all relatively young binaries, even those which are on the main sequence and appear normal. For example, the unexpected photometric complications found by HALL and HUBBARD (1971) in the detached $\mathrm{B} 4 \mathrm{~V}+\mathrm{A} 4 \mathrm{~V}$ eclipsing binary HS Herculis, can be understood, not in terms of post-mainsequence evolution, but as a consequence of its youth.

\section{Adknowledgement}

The National Science Foundation generously supported this work through Research Grants GP 8084 and GP 19895 and International Travel Grant GP 30029.

References:

BATTEN, A. H., 1970, P.A.S.P. 82, 574.

BERLAGE, H. P., 1968, The Origin of the Solar System (Oxford: Pergamon Press), p. 64.

BODENHEIMER, P., and OSTRIKER, J. P., 1970, Ap. J. 161, 1101.

DEVINNEY, E. J., Jr., HALL, D. S., and WARD, D. H., 1970, P.A.S.P. 82, 51.

DOREMUS, C., 1970, P.A.S.P. 82, 745.

HALL, D. S., and GARRISON, L. M., Jr., 1969, P.A.S.P. 81, 771 (Paper I).

HALL, D. S., and HUBB ARD, G. S., 1971, P.A.S.P. 83, in press.

HARRIS, D. L., III, 1968, in Basic Astronomical Data, K. Aa. Strand, ed. (Chicago: University of Chicago Press), p. 269.

HUANG, S. S., 1963, Ap. J. 138, 342.

HUANG, S. S., 1965 Ap. J. 141, 976.

IBEN, I., 1965, Ap. J. 141, 993.

IBEN, I., 1967, Annual Review of Astronomy and Astrophysics 5, 571.

JOHNSON, H. M., 1965, Ap. J. 142, 964.

LARSON, R. B., 1969, M. N. 145, 271.

MARKS, D. W, and CLEMENT, M. J., 1871, Ap. J. 1C6, L.27.

MERRILL, J. E., 1953, Princeton Obs. Contr. No. 23, p. 370.

MURPHY, R. E., 1971, preprint.

PARENAGO, P. P., 1947, Peremennye Zvezdy 6, 217.

PARSONS, S. B., 1971, preprint.

PLAVEC, M., 1068, in Advances in Astronomy and Astrophysics 6, Z. Kopal, ed. (New York: Academic Press), p. 216.

PLAVEC, M., 1970, P.A.S.P. 82, 957.

PLAVEC, M., and KRATOCH VIL, P., 1964, Bull. Astr. Inst. Czechoslovakia 15, 165.

RUSSELL, $H$. N., and MERRILL, J. E., 1952, Princeton Obs. Contr. No. 26, p. 44.

SHARPLESS, S., 1966, in Vistas in Astronomy, Vol. 8, A. Beer and K. Aa. Strand, eds. (Oxford: Pergamon Press), p. 127.

STRUVE, O., and TITUS, J., 1944, Ap. J. 99, 84.

WALKER, M. F., 1969, Ap. J.' 155, 447. 
Table 1: Unrectified Folded Normal Points

\begin{tabular}{|c|c|c|c|c|c|}
\hline \multirow[t]{2}{*}{$\Theta$} & \multirow[t]{2}{*}{ lv } & $16: 74$ & 0.876 & $132 \% 91$ & 1.003 \\
\hline & & $17^{\circ} .21$ & 0.893 & $151 \% 99$ & 1.001 \\
\hline 0.26 & 0.555 & $18 \% 29$ & 0.936 & $153 \% 90$ & 1.005 \\
\hline 0.72 & 0.556 & $18 \% 68$ & 0.962 & $156^{\circ} .71$ & 1.002 \\
\hline $1 \% 04$ & 0.556 & $19{ }^{\circ} 98$ & 0.990 & $158^{\circ} 11$ & 0.997 \\
\hline 1966 & 0.557 & $20 \% 92$ & 0.972 & $158^{\circ} .90$ & 0.994 \\
\hline 3.02 & 0.543 & 21974 & 0.961 & $160 \% 38$ & 0.978 \\
\hline $4: 60$ & 0.536 & $22 \div 75$ & 0.963 & $163: 15$ & 0.966 \\
\hline $5 ? 22$ & 0.557 & $23 \div 47$ & 0.968 & $167^{\circ} \cdot 11$ & 0.975 \\
\hline $6{ }^{\circ} 34$ & 0.574 & $23: 87$ & 0.980 & $171{ }^{\circ} 50$ & 0.964 \\
\hline $7^{\circ}{ }^{52}$ & 0.560 & $24 \div 59$ & 0.976 & 174935 & 0.976 \\
\hline 8.18 & 0.556 & $26: 06$ & 0.960 & $177^{\circ} .55$ & 0.966 \\
\hline 8.54 & 0.557 & $27^{\circ}: 04$ & 0.957 & 179.68 & 0.957 \\
\hline $9 \% 10$ & 0.562 & $28 \stackrel{\circ}{19}$ & 0.964 & 0.79 & 0.488 \\
\hline $9 \div 54$ & 0.554 & $28 \div 98$ & 0.965 & $\Theta$ & $l_{B}$ \\
\hline $10^{0} 34$ & 0.573 & 30.38 & 0.961 & 2916 & 0.488 \\
\hline $10 \% 90$ & 0.609 & 31.72 & 0.953 & $27 \div 90$ & 0.965 \\
\hline $11 \%_{38}$ & 0.615 & $32 \% 87$ & 0.969 & 29.63 & 0.957 \\
\hline $11 \% 92$ & 0.633 & $34: 31$ & 0.956 & 31.953 & 0.978 \\
\hline $12 \div 38$ & 0.654 & $38 \% 95$ & 0.970 & 130.07 & 1.001 \\
\hline $12 \% 70$ & 0.676 & 51.68 & 0.989 & $154 \% 87$ & 1.000 \\
\hline $13 \% 04$ & 0.698 & $58 \% 32$ & 0.971 & $177^{0_{30}}$ & 0.965 \\
\hline $130^{\circ} 6$ & 0.714 & $62 \div 57$ & 0.966 & 2927 & 0.443 \\
\hline $14: 14$ & 0.747 & 76.39 & 0.985 & $\Theta$ & $\mathrm{lu}_{\mathrm{U}}$ \\
\hline $14 \% 54$ & 0.776 & 88.67 & 1.002 & 28.58 & 0.951 \\
\hline $14: 94$ & 0.796 & $99^{\circ} 07$ & 0.988 & $30 \%_{42}$ & 0.945 \\
\hline $15: 34$ & 0.805 & $114^{\circ} .66$ & 0.993 & 156.02 & 1.000 \\
\hline $15: 80$ & 0.836 & 119.16 & 1.002 & $176^{\circ} .54$ & 0.974 \\
\hline $16 \%_{27}$ & 0.846 & $128 \% 09$ & 1.000 & $179^{\circ} 53$ & 0.950 \\
\hline
\end{tabular}

Table II: Luminosities at Maxima and Minima

$\begin{array}{lccc} & \mathrm{lv} & \mathrm{l}_{\mathrm{B}} & \mathrm{l}_{\mathrm{U}} \\ \text { primary minimum } & 0.556 & 0.488 & 0.443 \\ \text { primary shoulder } & 0.963 & 0.967 & 0.948 \\ \text { secondary shoulder } & 1.000 & 1.000 & 1.000 \\ \text { secondary minimum } & 0.963 & 0.965 & 0.962\end{array}$

Table III : Relative Luminosities based on $\mathrm{f}_{\mathrm{B}}=0.55$

$\begin{array}{lcccc} & \mathrm{LV}_{V} & \mathrm{~L}_{\mathrm{B}} & \mathrm{LU}_{\mathrm{U}} & \mathrm{V} \\ \mathrm{B} 3 & 0.74 & 0.87 & 0.92 & 8 \mathrm{~m} 3 \\ \text { disk (facing) } & 0.26 & 0.13 & 0.08 & 9 m 4 \\ \text { disk (opposite) } & 0.22 & 0.10 & 0.03 & 9 m 6\end{array}$

Table IV: Parameters for the three Cases considered

$\begin{array}{ccccc}\text { Case } & \text { i } & \mathrm{h} & \mathrm{f}_{\mathrm{D}} & \Delta \mathrm{l}_{\mathrm{i}}^{\text {prt }} / \Delta \mathrm{l}_{0^{\text {prl }}} \\ \text { I } & 90^{\circ} & 0.07 & 0.32 & 1.00 \\ \text { II } & 87^{\circ} & 0.08 & 0.28 & 1.03 \\ \text { III } & 84^{\circ} & 0.16 & 0.14 & 1.06\end{array}$


Table V: Absolute Dimensions

\begin{tabular}{|c|c|}
\hline B 3 Primary & Disk-Shaped Secondary \\
\hline Mass: $5 \mathfrak{M}_{\odot} \pm 1 \mathfrak{M}_{\odot}$ & Mass: $2 \mathfrak{m}_{\odot} \pm 1 \mathfrak{M}_{\odot}$ \\
\hline Radius: $2.5 \mathrm{R}_{\odot} \pm 0.3 \mathrm{R}_{\odot}$ & Equatorial Radius: $7.5 \mathrm{R}_{\odot} \pm 0.5 \mathrm{R}_{\odot}$ \\
\hline$M_{v}:-o m 7 \pm o m 3$ & $\begin{aligned} \text { Total Thickness: } & 3 \mathrm{R}_{\odot} \\
M_{V} \text { (facing): } & +0 \mathbf{m}_{4} \\
\text { (opposite) }: & +0 \mathbf{o m}_{6}\end{aligned}$ \\
\hline
\end{tabular}

Table VI: Times of Primary Minimum

$\begin{array}{clcrc}\text { JD (hel.) } & \text { Source } & \text { Year } & \text { Cycle } & \text { O-C } \\ 2,422,717.28 & \text { GRAFF, HARTWIG } & 1921 & \mathbf{- 2 7 1 2} & \text { odoo } \\ 2,423,131.30: & \text { HAAS } & 1922 & -2648 & -0.09: \\ 2,423,830.18 & \text { GRAFF } & 1924 & -2540 & -0.03 \\ 2,423,843.20 & \text { HEISE } & 1924 & -2538 & +0.05 \\ 2,430,792.50: & \text { SCHNELLER } & 1943 & -1464 & +0.01: \\ 2,431,148.35: & \text { SCHNELLER } & 1944 & -1409 & -0.02: \\ 2,436,557.71 & \text { WALKER } & 1958 & -573 & -0.02 \\ 2,440,265.343 & \text { HALL } & 1969 & 0 & 0.000\end{array}$

Discussion to the paper of HALL

TINBERGEN: This system seems to be a prime candidate for spectropolarimetry. It should be possible to predict in detail the polarization to be expected, as a function of time and wavelength.

HALL: Yes, this would be a very good thing to do.

$\mathrm{KOCH}$ : Do I understand that this is an incipient triple system?

HALL: No, I am not thinking that. After all, the secondary is not shaped like a peanut. I rather suspect the disk will somehow evolve rather soon into a normal, spherical star.

BAKOS: Do you have a spectrum of the star outside the eclipse? You may be able to see the secondary in the distribution of the continuous spectrum.

HALL: In principle this is a very good idea and should be tried, but in practice it may be difficult since the secondary seems to be one or two magnitudes fainter than the B star. Attempts to detect the secondary in the infrared spectrum have so far failed.

QUESTER: There was some flicker during total phase. Does this flicker also occur during maximum?

HALL: Yes, the scatter also appears outside eclipse. It is difficult to decide for sure whether the scatter is real, because the observations were very difficult to make. But there is some indication that the light curve is intrinsically variable. Indeed it would not be surprising to find a disk-shaped object such as this sowewhat unstable. For this reason it would be very good to obtain a few light curves.

BEHR: How did you correct your observations for sky background?

HALL: I offset every time to the same part of the nebulosity, a part which was an equivalent distance from the nearest two bright stars. And although this part of the nebula might have been a little brighter or fainter than the part surrounding BM Ori, the difference could have caused an overall shift upward or downward in the light curve of only about 0.01 or 0.02 luminosity units, which is not very important. 